\title{
Immuno-dermatological processes involved in chronic skin diseases: Highlights of the Second Conference of the Romanian Society for Immuno-Dermatology, Bucharest, September, 2018
}

\author{
DANIEL BODA $^{1}$ and CRISTINA DEHELEAN ${ }^{2}$ \\ ${ }^{1}$ Dermatology Research Laboratory, 'Carol Davila' University of Medicine and Pharmacy, 050474 Bucharest; \\ ${ }^{2}$ Faculty of Farmacy, 'Victor Babes' University of Medicine and Pharmacy, 300041 Timisoara, Romania
}

Received March 19, 2019; Accepted June 19, 2019

DOI: $10.3892 / \mathrm{etm} .2019 .7690$

Immunological phenomena have been shown to be extensively involved in recent years as key pathways in the pathogenesis and outcomes of several chronic skin conditions and have been linked to novel therapeutic options. Thus, this special issue focuses on reviews, original articles and case presentations in immuno-dermatology, in order to partially elucidate those pathways and processes.

The main topics covered in this special issue were immunological pathways involved in the pathogenesis of psoriasis, lichen rubra, atopic dermatitis, chronic urticaria, pruritus and adverse drug reactions.

Polymorphisms of the IL-17 family have been shown to be associated with the severity and response to treatment in psoriasis, and also with the risk of inflammatory, infectious, autoimmune or neoplastic pathologies. The significant difference in the presence or absence of susceptibility loci in different population is due to genetic background and environmental factors that have a major impact on disease predisposition. Puşcaş et al (1) reviewed the importance and influence of the IL-17 polymorphisms as predictors of response to treatment and severity of the disease.

Guo et al (2) aimed their study to assess the effects of the traditional Chinese medicine (TCM), white mange mixture, in a murine model of vaginal psoriasis, proving that white mange mixture can significantly inhibit vaginal psoriasis in a mouse model by decreasing the amount of epithelial KC cell PCNA and the production of the inflammatory cytokine, GM-CSF in serum.

Morar et al (3) performed an immunohistochemical analysis of psoriatic plaques and perilesional skin in patients

Correspondence to: Dr Daniel Boda, Dermatology Research Laboratory, 'Carol Davila' University of Medicine and Pharmacy, 8 Eroii Sanitari Avenue, 050474 Bucharest, Romania

E-mail: danielboday@yahoo.com

Key words: chronic skin diseases, immuno-dermatology with psoriasis vulgaris, aiming to investigate the expression of tumor necrosis factor- $\alpha$ (TNF- $\alpha$ ), vascular endothelial growth factor receptor-2 (VEGFR-2) and prolactin receptor (PRLR) in psoriatic skin and to evaluate the association with disease severity. Positive cells for VEGFR-2 and PRL were identified in the basal layer keratinocyte cells (VEGFR-2), sweat glands and hair outer shaft sheath (PRLR), without significant differences between the two types of samples. Their findings confirm the importance of TNF- $\alpha$ in psoriasis pathogenesis and a positive association with lesion severity. No significant differences were found for VEGFR-2 and PRLR; however, additional studies are required to establish their role.

Raducan et al (4) presented the outcome of biological anti-TNF- $\alpha$ treatment in severe psoriasis associated with chronic hepatitis $\mathrm{B}$, aiming to emphasize that the use of etanercept in this comorbidity of psoriasis is a successful therapeutic alternative, which may be safely used concomitantly with entecavir, with regular monitoring of viral load and hepatic function tests.

Boca et al (5) assessed, through a questionnaire, demographic criteria, as well as patient-related issues in patients with psoriasis in Romania, with questions about the prevalence, social impact and comorbidities of this chronic skin condition.

Though biological treatment is nowadays a key standard in moderate to severe plaque psoriasis, methotrexate is still used in countries with no access to costly treatments. Nedelcu et al (6) summarized in a review the efficacy of methotrexate as an antiinflammatory and anti-proliferative drug in dermatology.

Biosimilars are new drugs, highly similar copies of biological medicines, equally effective and safe, but are available at lower prices. Constantin et al (7) provide a brief review of the current status of biosimilars approved in the European Union for dermatological use.

Ilie et al (8) summarized in a review the effects of capsaicin on neurogenic inflammation and nociception, as well as its potential diagnostic value and therapeutic impact in various conditions involving the impairment of sensory nerve fibers.

Rusu et al (9) performed a review on probiotics in atopic dermatitis, showing that there was an increase in intestinal permeability in patients with atopic dermatitis and also a 
reduction in cutaneous microbiome diversity. Probiotics modulate the general microbiome and immune status by improving the intestinal barrier; these effects may be responsible for reducing allergic phenomenon and atopic dermatitis severity. They structured the results by age group, such as infants, 1-18 year olds, adults, and also by pregnancy and lactation.

Satureja hortensis L. has gained increased interest due to its chemical composition. It is found abundantly in monoterpenes, aglyconic and glycosylates flavonoids, and phenolic acids, which leads to an important biological activity. Popovici et al (10) compared the biological activity of volatile oil (VO) and total hydro alcoholic extract (TE) of Satureja hortensis L. in terms of: i) antioxidant activity; ii) antimicrobial activity; and iii) viability, migration and proliferation on two healthy cell lines and two melanoma cell lines.

Nwabudike et al (11) presented four cases of generalized lichen planus (LP) with and without mucosal involvement treated homeopathically, showing that in its long-standing, generalized form, with mucosal involvement, LP may respond to individualized homeopathy, concluding that more research may clarify the place of homeopathy in LP therapy.

$\mathrm{Xu}$ et al (12) studied the effects of lidocaine on the safety of postoperative skin reconstruction following malignant melanoma resection, showing that the local application of lidocaine can promote wound healing to a certain extent, reduce pain, and promote postoperative skin reconstruction.

While recent articles have focused on the positive uses of $\beta$-blockers, it may also be wise to focus our attention on the potential dermatologic adverse effects that may follow $\beta$-blocker use, as well as possible therapeutic approaches to these. Tatu et al (13) focused on those dermatoses resulting from $\beta$-blocker use, which have an immunologic basis.

Bumbacea et al (14) analyzed 2 severe cases of cold urticaria and symptomatic dermographism with a completely different response to omalizumab, concluding that although many clinical studies support the use of omalizumab in the treatment of patients with this condition, we certainly need more data for prediction of a good clinical response.

$\mathrm{Hu}$ et al (15) aimed their study in investigating the clinical features and possible influencing factors of pruritus in patients with chronic renal failure proving a significant difference in the levels of serum urea nitrogen, creatinine, serum phosphorus, calcium x phosphorus, and PTH (parathyroid hormone) between patients with pruritus and non-pruritus. They also showed that local cold and heat stimulation can relieve pruritus to some extent, and adequate hemodialysis can also relieve itching as well as reducing xeroderma.

Linte et al (16) present the case of an 11-year-old child with cachexia, severe dextroscoliosis, pectus carinatum, secondary restrictive pulmonary disease, and an incidental finding of a large ostium secundum atrial septal defect (ASD) that was identified on preoperative assessment for surgical correction of the spinal deformity.

To conclude, the quality of the articles submitted to the Special Issue of 'Experimental and Therapeutic Medicine' meets the Journal's standards, providing fundamental scientific points, thus achieving the proposed aims of the issue. We would like to take this opportunity and thank all the authors for their valuable contribution.

\section{References}

1. Pușcaș AD, Cătană A, Pușcaș C, Roman II, Vornicescu C, Șomlea $M$ and Orăsan RI: Psoriasis: Association of interleukin-17 gene polymorphisms with severity and response to treatment (Review). Exp Ther Med 18: 875-880, 2019.

2. Guo J and Liu J: Effect of white mange mixture in a murine model of psoriasis. Exp Ther Med 18: 881-887, 2019.

3. Morar II, Tabăran FA, Mocan T, Jianu EM, Orăsan MS, Pop AD and Orăsan RI: Immunohistochemical study of psoriatic plaques and perilesional skin in psoriasis vulgaris patients: A pilot study. Exp Ther Med 18: 888-894, 2019.

4. Raducan A, Bucur S, Caruntu C, Constantin T, Nita IE, Manolache $\mathrm{N}$ and Constantin MM: Therapeutic management with biological anti-TNF- $\alpha$ agent in severe psoriasis associated with chronic hepatitis B: A case report. Exp Ther Med 18: 895-899, 2019.

5. Boca AN, Ilies RF, Vesa S, Pop R, Tataru AD and Buzoianu AD: The first nation-wide study revealing epidemiologic data and life quality aspects of psoriasis in Romania. Exp Ther Med 18: 900-904, 2019

6. Nedelcu RI, Balaban M, Turcu G, Brinzea A, Ion DA, Antohe M, Hodorogea A, Calinescu A, Badarau AI, Popp CG, et al: Efficacy of methotrexate as anti-inflammatory and anti-proliferative drug in dermatology: Three case reports. Exp Ther Med 18: 905-910, 2019.

7. Constantin MM, Cristea CM, Taranu T, Bucur S, Constantin T, Dinu A, Jinga $M$ and Nita IE: Biosimilars in dermatology: The wind of change (Review). Exp Ther Med 18: 911-915, 2019.

8. Ilie MA, Caruntu C, Tampa M, Georgescu SR, Matei C, Negrei C, Ion RM, Constantin C, Neagu M and Boda D: Capsaicin: Physicochemical properties, cutaneous reactions and potential applications in painful and inflammatory conditions (Review). Exp Ther Med 18: 916-925, 2019.

9. Rusu E, Enache G, Cursaru R, Alexescu A, Radu R, Onila O, Cavallioti T, Rusu F, Posea M, Jinga M and Radulian G: Prebiotics and probiotics in atopic dermatitis (Review). Exp Ther Med 18: 926-931, 2019.

10. Popovici RA, Vaduva D, Pinzaru I, Dehelean CA, Farcas CG, Coricovac D, Danciu C, Popescu I, Alexa E, Lazureanu V and Stanca HT: A comparative study on the biological activity of essential oil and total hydro-alcoholic extract of Satureja hortensis L. Exp Ther Med 18: 932-942, 2019.

11. Nwabudike LC, Miulescu M and Tatu AL: Case series of an alternative therapy for generalised lichen planus: Four case studies. Exp Ther Med 18: 943-948, 2019.

12. Xu K, Wang J, Yang L, Wan L and Wang Y: Effect of lidocaine on the safety of postoperative skin reconstruction after malignant melanoma resection. Exp Ther Med 18: 949-954, 2019.

13. Tatu AL, Elisei AM, Chioncel V, Miulescu M and Nwabudike LC: Immunologic adverse reactions of $\beta$-blockers and the skin (Review). Exp Ther Med 18: 955-959, 2019.

14. Bumbăcea RS, Deaconu CG and Berghea EC: Management problems in severe chronic inducible urticaria: Two case reports. Exp Ther Med 18: 960-963, 2019.

15. Hu T, Wang B, Liao X and Wang S: Clinical features and risk factors of pruritus in patients with chronic renal failure. Exp Ther Med 18: 964-971, 2019.

16. Linte A, Balanescu AR, Onorato E, Balanescu DV, Bojinca VC, Drakonaki E, Bojinca $M$ and Balanescu SM: Percutaneous closure of a large atrial septal defect in a child with severe dextroscoliosis: A case report. Exp Ther Med 18: 972-975, 2019.

This work is licensed under a Creative Commons Attribution-NonCommercial-NoDerivatives 4.0 International (CC BY-NC-ND 4.0) License. 